\title{
Rehabilitation in Squamous Cell Carcinoma of Lateral Border of the Tongue - A Case Report
}

\author{
Diksha Nagrale $^{1}$, Pankhuri Multani ${ }^{2}$, Anagha Armarkar ${ }^{2}$, Amrita Sakharwade $^{3}$ \\ ${ }^{1}$ Professor, Department of Community Based Rehabilitation, Smt. Radhikatai Pandav College of Physiotherapy, \\ Nandanvan, Nagpur, India \\ ${ }^{2}$ Professor, Smt. Radhikatai Pandav College of Physiotherapy, Nandanvan, Nagpur, India. \\ ${ }^{3}$ Assistant Professor, Smt. Radhikatai Pandav College of Physiotherapy, Nandanvan, Nagpur, India. \\ Corresponding Author: Diksha Nagrale
}

DOI: https://doi.org/10.52403/ijhsr.20220316

\begin{abstract}
Oral cancer is the world's sixth most prevalent cancer, with oral squamous cell carcinoma accounting for the vast majority of occurrences (OSCC). The most prevalent intraoral head and neck cancer is squamous cell carcinoma (SCC) of the tongue. It is the second most common cancer in India, after SCC of the lip and oral cavity, with a male incidence of 13.9 per cent compared to 4.3 per cent in females. SCC of the tongue is quite uncommon until the age of 60. The use of smoking and smokeless cigarettes, areca nut use, and alcohol consumption are the main risk factors for oral SCC. A patient experienced the loss of motion, spasms, weakness, and function following tongue cancer surgery. Buccal mucosa SCC is a malignant tumour of the oral cavity that is aggressive. It's associated with a high rate of regional recurrence, with the inner cheek lining, lateral border of the tongue, oropharynx, mouth floor, and lips being the most commonly affected areas. The most common postoperative oral problems that occur after radiation and chemotherapy are mucositis, infection, pain, bleeding, difficulty swallowing, injury to the glands that produce saliva (xerostomia) or damage to the muscles and joints of the jaw and neck (trismus), loosening of teeth, difficulty wearing dentures, painful swallowing (odynophagia), speech impairment (dysarthria), and development of a neck mass as a sign of lymph node metastasis.
\end{abstract}

Conclusion: Following carcinoma of the lateral border of the tongue, physical therapy included exercises, spasm reduction, Kinesio taping, manual therapy, and patient education.

Keywords- Squamous cell carcinoma, Glossectomy, Tongue, Physiotherapy Rehabilitation.

\section{INTRODUCTION}

Buccal mucosa squamous cell carcinoma is a rare but aggressive kind of oral cavity cancer. It's also linked to a high probability of local-regional recurrence, and it's more likely in persons who chew tobacco and/or smoke, whether or not they drink alcohol. It is vital to highlight that approximately $4 \%-10 \%$ of these occurrences are unrelated to the risk variables. Other variables may be involved in some cases of OSCC, such as infectious agents like the human papillomavirus, immunological abnormalities or immunosuppression, issues with carcinogen metabolism, or errors in DNA repair enzymes (1). At the median age of 61 years, SCC of the tongue is discovered. Though SCC of the tongue is more common in males than girls around the world, recent European research over the last decade has shown a rising tendency of SCC of the tongue in females (2). In about 45 per cent to 48 per cent of instances, oral cancer appears as ulcers or ulcerated tumours. The diagnosis of oral ulcerative lesions can be difficult due to the vast range of causal variables and unpredictable presenting 
symptoms. These carcinomatous ulcers can be mistaken for other clinical entities at first, and are frequently misdiagnosed as a nonneoplastic ulcerative lesions. Because of their chronicity and unresponsiveness to numerous therapies, lesions lasting two weeks or longer often pose a problem (3). Dental rehabilitation is an important part of such complete treatments, as it aims to replace teeth that have been lost or are missing as a result of tumour excision (4). Depending on the stage of the disease and the patient's state, reconstructing oral cavity deformities is always a difficult task. Because of this unique and complex threedimensional anatomical context, reconstructive surgeries frequently result in a compromise that results in functional morbidity and, more importantly, may limit the likelihood of appropriate prosthetic rehabilitation (4). The surgical excision of cancer often leaves the patient with significant functional impairment. Aspiration has been described as such a debilitating consequence that supplementary total laryngectomy for individuals undergoing total glossectomy has been recommended (5). Aspiration is believed to be such a debilitating consequence that supplementary total laryngectomy for individuals undergoing total glossectomy has been recommended (5). Patients with large tumours often have a degree of tongue immobility at first, which has triggered their adaptation to the aspiration mechanism (5). Those exposed to the equivalent of 0 to 19 cigarettes and 0 to 2 whiskey equivalents per day for at least 30 years had a 3.9-fold increased risk of multiple primary cancer than those exposed to the equivalent of 0 to 19 cigarettes and 0 to 2 whisky equivalents per day. A lack of fresh fruits and vegetables in one's diet, infectious agents like candida or viruses, immune weakness, and sun exposure are all probable reasons. Various combinations of radiotherapy and surgery have been used to treat carcinoma of the base of the tongue. The necessity to establish normal physiologic features makes resection of this area a critical difficulty in reconstruction (6). Men are more likely than women to be in their sixties and seventies. The mucosa buccally, the alveolar crest of the jaw, the tongue, and the gingiva are the most commonly affected intraoral locations (7). Regional lymph nodes are painful and swollen in verrucous carcinoma due to involvement, simulating metastatic tumours, although lymph nodes were not present in our patients (7). The primary course of treatment for verrucous cancer is surgery. The patient, in this case, was treated by surgical excision. Radiotherapy has the potential to transform the tumour into a squamous cell carcinoma, which has a bad prognosis. (7). An oral verrucous carcinoma is a clinicopathological form of oral squamous cell carcinoma. The buccal mucosa and alveolus, as well as the gingiva, are the most commonly impacted intraoral locations. It's a locally aggressive tumour. It is generally linked to tobacco use, particularly chewed and snuffed kinds, and is hence known as "snuff dipper's cancer." Because of the dysplastic cells around the tumour, recurrence is common (7). Total glossectomy may be recommended in patients who have severe illness or have failed to respond to conventional treatments. Patients usually experience persistent pain, speech difficulties, and deglutition at this stage. In the first two phases of deglutition, the tongue plays a critical function. The bolus remains a passive, inert, and adynamic conduit despite reconstructive treatments that keep it flowing from the lips to the throat. Unless protective mechanisms are established, this could result in aspiration pneumonitis and lung abscess (2). The physical treatment programme included exercises, spasm reduction, Kinesio taping, manual therapy, and patient education (5).

\section{CASE DESCRIPTION}

A 58-year-old woman appeared to be fine two months ago when she developed a painful non-healing ulcer on her lower lip that began small and steadily grew to its current size. She had a history of related pain that began gradually, was dull hurting, 
intermittent, and radiating in nature, and was aggravated by mastication and relieved by medicine. There is no history of trauma. There is a history of burning sensation on the consumption of hot and spicy food for 2 months. There is a change in the consistency of saliva for 15 days.

Past History: The patient was diagnosed with well-differentiated squamous cell carcinoma of the tongue in Biopsy on $11 / 2 / 2020$ and underwent partial glossectomy with Modified radical neck dissection on 17/3/2020 in a local hospital. The patient has a history of tobacco chewing 3-4 times a day for 8 yrs.

Drug History: The patient is not allergic to any medication taken by her until now.

Family History: No significant family history.

Personal History:

Diet: Vegetarian

Sleep-wake cycle: Normal

Appetite: adequate

Bowel and bladder: Normal

Menstrual History: Menopause attained 8 yrs back.

Clinical findings: The patient in the supine position was examined with both shoulders at the same level. On physical examination, vital signs including temperature were normal, pulse rate 92 beats/min, RR-23 breaths/min, BP-130/90mmhg. The cardiovascular and respiratory system was seen to be normal during the general examination. The pain was calculated using the Numerical Pain Rating Scale (NPRS) on which the patient marked $4 / 10$ during rest and $7 / 10$ during movement.

Temporomandibular Joint- mouth opening- 4 finger

Tongue mobility- Restricted. cavity.

No obvious growth in the oral

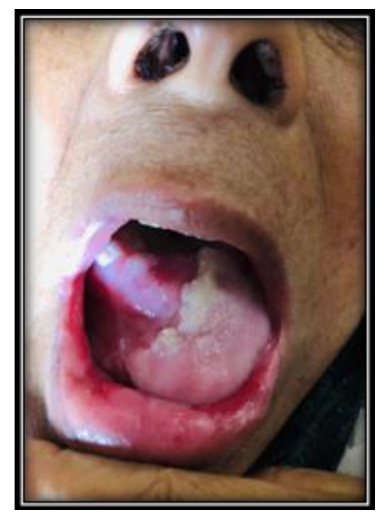

Fig. 1- Showing Squamous cell carcinoma on lateral border of the tongue.

\section{Physiotherapy Intervention:}

Patients with oral cancer who are undergoing therapies such as head and neck exercises, mouth opening exercises with TheraBite devices, and shoulder mobility can benefit from physiotherapy. It helps to prevent and/or treat a variety of side effects that can occur as a result of cancer treatment. Physiotherapy rehabilitation programmes help cancer survivors restore functional range of motion (ROM) and so improve their quality of life, especially after surgery (1). TENS is a physiotherapeutic approach that is frequently used to treat pain associated with cancer and its treatments.
(1). It provides a strong, non-painful sensation that lasts for a long time. TENS can be used to relieve postoperative pain symptoms at the surgical site by using a low-frequency continuous mode and changing the intensity that the patient can tolerate for a period based on the pain level as described above. TENS is an effective therapy for reducing pain in cancer patients and aiding in the improvement of joint ROM in cancer survivors (1). Patients with certain disabilities were treated with alternative physiotherapeutic procedures to reduce discomfort and improve their quality of life. Muscle stretching, strengthening 
exercises, chest physiotherapy, mobility exercises, and other motions have been recognised as the most effective rehabilitation approaches. Physical therapy has been shown to help relax muscles, increase joint flexibility, reduce fatigue, and improve overall physical and functional well-being, as well as increase awareness of altered posture, walking, and breathing patterns, manage swallowing and mouth opening difficulties, and improve overall physical and functional well-being. The clinical consequences of physiotherapy treatment strategies in palliative care to reduce cancer pain have been thoroughly studied. Physiotherapy treatment has been demonstrated to help cancer patients with symptom reduction and improved quality of life. According to physiotherapy in postoperative problems of head and neck malignancies, multidisciplinary rehabilitation should be made an integral element of the overall management of head and neck cancer survivors (1). Our approach to speech therapy has been to first examine the patients to see what they are capable of. $\mathrm{P}, \mathrm{B}$, and $\mathrm{M}$ are examples of labial sounds that are generally maintained. Otherwise, additional consonants and vowels are deleted or altered, reducing intelligibility significantly. Other accessory structures, including the lips, lower jaw, soft palate, uvula, and buccal muscle, adopt exaggerated postures to help with the new articulation mechanics. (5).

\section{RESULTS}

The current case is a very unusual case of advanced tongue carcinoma in a 58year-old woman. Because SCC of the tongue is becoming more common, steps should be done to organise and implement tobacco and alcohol abuse cessation programmes. The above studies conclude that physiotherapy rehabilitation led to enhancement in functional goals of the patient which stand a major understanding towards a successful recovery.

\section{DISCUSSION}

Physiotherapy treatment has been found to benefit cancer patients in terms of symptom alleviation and quality of life. Interdisciplinary rehabilitation should be incorporated into the overall management of cancer survivors, according to physiotherapy in postoperative complications of tongue carcinoma (1). SCC of the tongue is more common in men, with a predominance in the sixth to seventh decades of life, and the etiological factors are unknown. The most common risk factors, such as smoking and drinking, are more common in the senior population than in the younger population. (2). One of the most malignant subtypes of intraoral head and neck tumours is carcinoma tongue. (2). Advanced tongue carcinoma has a dismal prognosis. Despite the completion of all treatment methods, the prognosis is poor in this situation, and the risks of recurrence are higher. Tumour crossing the midline, cervical lymph node-positive with extranodal extension, lymph vascular invasion, residual tumour, recurrence, and surgical resection completeness are some of the independent factors that determine the prognosis of the disease. In this situation, long-term follow-up is critical. We've also kept her on a regular follow-up schedule to avoid any recurrences. (2).

\section{CONCLUSION}

The above study suggests that early rehabilitation of physiotherapy contributed to the improvement of the patient's functional objectives, which are a significant understanding of a good recovery.

Author's Contribution: For the concept, assessment and evaluation, data collection analysis and interpretation of the data, each author made the best contribution.

Informed Consent: Proper consent was taken from the patient's son for writing the case report. 
Funding: There are no financial conflicts of interest to disclose.

Conflict Of Interest: Authors have no conflicts of interest to disclose.

\section{Acknowledgement: None}

\section{REFERENCES}

1. Pattanshetty R, Mathias O. Postoperative physiotherapy management for complications related to cancer of buccal mucosa (head and neck cancer). Indian $\mathbf{J}$ Phys Ther Res. 2019;1(2):126.

2. Kadam SS, Akshay Deshpande, Sonawane S, Tejaswini Kadam. Advanced Carcinoma of Tongue in a 38-Year-Old Female: A Case Report. 2020 [cited 2022 Jan 22].

3. Keerthi Narayan V. Verrucous Carcinoma of Buccal Mucosa in Female: A Rare Case Report of Traumatic Tooth Origin. Marconi G, editor. Case Rep Dent. 2021 Jun 11;2021:1-5.

4. Romeo U, Lollobrigida M, Palaia G, Laurito D, Cugnetto R, De Biase A. Soft Tissue Management and Prosthetic Rehabilitation in a Tongue Cancer Patient. Case Rep Dent. 2013;2013:1-5.

5. Effron MZ, Johnson JT, Myers EN, Curtin H, Beery Q, Sigler B. Advanced Carcinoma of the Tongue: Management by Total Glossectomy Without Laryngectomy. Arch Otolaryngol - Head Neck Surg. 1981 Nov 1;107(11):694-7.

6. Schechter GL, Sly DE, Roper AL, Jackson RT, Bumatay J. Set-Back Tongue Flap for Carcinoma of the Tongue Base. Arch Otolaryngol - Head Neck Surg. 1980 Nov 1;106(11):668-71.

7. Eltohami YI, Alim NE, Suleiman AM, Abuaffan AH. Snuff (Tomback) Dipping Verrucous Carcinoma: A Case Report. otolaryngology [Internet]. 2017 [cited 2022 Jan 22];07(02).

How to cite this article: Nagrale D, Multani P, Armarkar A et.al. Rehabilitation in squamous cell carcinoma of lateral border of the tongue - a case report. Int J Health Sci Res. 2022; 12(3): 123-127. DOI: https://doi.org/10.52403/ijhsr. 20220316 